\title{
Secondary genetic factors in cystic fibrosis lung disease
}

Cystic fibrosis (CF) is the most common autosomal recessive genetic disorder amongst populations of northern European descent. Cloning of the cystic fibrosis transmembrane regulator (CFTR) gene in $1989^{1}$ has allowed the assessment of clinical phenotype in patients with a specific genetic abnormality. Genotype/phenotype analysis showed a good correlation between the common CF mutation (deletion of phenylalanine at position 508 in the CFTR gene) and pancreatic insufficiency. This correlation does not hold for pulmonary disease which can vary markedly between patients with the same $\Delta$ F508 CFTR mutation. ${ }^{2}$ Many explanations have been advanced to account for this finding. Pulmonary disease is influenced by environmental factors such as the patient's age, pancreatic status, treatment regime, social class, smoking history, nutritional status, and colonisation with strains of Pseudomonas aeruginosa or Burkholderia cepacia. ${ }^{3-8}$ Moreover, those patients who receive care in a dedicated CF centre have better lung function when all the known environmental factors are taken into account. ${ }^{9}$

There is also evidence that the severity of pulmonary disease in CF is linked to other genetic factors outside the CFTR gene locus. Support for this comes from consortium and family studies ${ }^{2}{ }^{10}$ and from the analysis of mice in whom the CFTR gene has been deleted. ${ }^{11}$ Investigators have focused on genes involved in host defence and inflammation. Polymorphisms that result in high levels of antipseudomonas $\mathrm{IgG}_{3},{ }^{12}$ high levels of tumour necrosis factor (TNF) $\alpha,{ }^{13}$ low levels of glutathione $S$-transferase $M 1,{ }^{13}$ and low levels of mannose binding protein ${ }^{14}{ }^{15}$ have all been associated with more severe lung disease in patients with CF. The risk of colonisation with $P$ aeruginosa is increased in CF patients with the class II DR7 allele, ${ }^{16}$ autoantibodies to bactericidal/permeability increasing protein, ${ }^{17}$ and with mild deficiency phenotypes of $\alpha_{1}$-antitrypsin. ${ }^{18}$ In our study these deficiency phenotypes were associated with better, rather than worse, lung function. ${ }^{19}$

It is on this background that Arkwright et al report their findings in this issue of Thorax. ${ }^{20}$ They have chosen to assess polymorphisms in the transforming growth factor $\beta$ (TGF- $\beta$ ) gene in 171 patients with the same CFTR mutation from three centres in Manchester, UK. The rationale for the study is that individuals who have polymorphisms in codon 10 and 25 express high levels of TGF- $\beta$ and this has been associated with more severe lung fibrosis following lung transplantation and in animal models of radiation and drug induced pulmonary disease. The authors found an interesting association between a mutation in codon 10 and more rapid decline in lung function in patients with $\mathrm{CF}$. The study took into account CF genotype and the patient's age but failed to correct for other factors such as nutritional status, smoking history, or the paediatric CF centre where the children received their care. These are important variables as they can introduce bias into the analysis. Ideally, studies should be designed to remove known confounding factors at the outset or should attempt to correct for these factors using statistical models and multivariate analysis.

The assessment of genetic polymorphisms using this type of observational study is of value in understanding disease mechanisms, but caution should be exercised in interpreting the results. They are subject to the bias that resides in relatively small numbers of heterogeneous patients and only modest effects on lung function. Only when replicated by other groups and supported by in vivo data from the lungs of patients with CF do any of the reported associations have real meaning. The goal of establishing why some patients with CF have a more rapid decline in lung function is vital as it will allow the identification of a subset of patients who require more intensive medical care. Moreover, it will enable us to determine pathways that modify the effect of the CFTR mutation. Further studies of the proposed genetic factors are now required, ideally by a consortium, with sufficient numbers of patients to correct for confounding environmental and genetic variables.

RAVI MAHADEVA DAVID A LOMAS

Respiratory Medicine Unit,

Department of Medicine,

University of Cambridge,

The Wellcome Trust Centre for Molecular Mechanisms in Disease,

Cambridge Institute for Medical Research,

Cambridge CB2 2XY,

UK

email:dall6@cam.ac.uk

1 Riordan JR, Rommens JM, Kerem B-S, et al. Identification of the cystic fibrosis gene: cloning and characterization of complementary DNA. Science 1989;245:1066-73.

2 The Cystic Fibrosis Genotype-Phenotype Consortium. Correlation between genotype and phenotype in patients with cystic fibrosis. $N$ Engl f Med 1993;329:1308-13.

3 Britton JR. Effects of social class, sex, and region of residence on age of death from cystic fibrosis. BMF 1989;298:483-7.

4 Gaskin K, Gurwitz D, Durie P, et al. Improved respiratory prognosis in patients with cystic fibrosis with normal fat absorption. 7 Pediatr $1982 ; 100$ : $857-62$

5 Campbell PW, Parker RA, Roberts BT, et al. Association of poor clinical status and heavy exposure to tobacco smoke in patients with cystic fibrosis tus and heavy exposure to tobacco smoke in patients with cystic fibrosis

6 Henry RL, Mellis CM, Petrovic L. Pseudomonas aeruginosa is a marker of poor survival in cystic fibrosis. Pediatr Pulmonol 1992;12:158-61.

7 Corey M, McLaughlin FJ, Williams M, et al. A comparison of survival, Corey $M$, McLaughlin FJ, Williams $M$, et al. A comparison of survival,
growth, and pulmonary function in patients with cystic fibrosis in Boston growth, and pulmonary function in patients with

8 Sun L, Jiang RZ, Steinbach S, et al. The emergence of a highly transmissable lineage of cbl+ Pseudomonas (Burkholderia) cepacia causing CF centre epidemics in North America and Britain. Nature Med 1995;1:661-6.

9 Mahadeva R, Webb K, Westerbeek RC, et al. Clinical outcome in relation to care in centres specialising in cystic fibrosis: cross sectional study. $B M \mathcal{F}$ 1998;316:1771-5.

10 Santis G, Osborne L, Knight RA, et al. Independent genetic determinants of pancreatic and pulmonary status in cystic fibrosis. Lancet 1990;336:1081-4.

11 Kent G, Iles R, Bear CE, et al. Lung disease in mice with cystic fibrosis. $\mathcal{F}$ Clin Invest 1997;100:3060-9.

12 Pressler T, Pandey JP, Espersen F, et al. Immunoglobulin allotypes and IgG subclass antibody response to Pseudomonas aeruginosa antigens in chronically infected cystic fibrosis patients. Clin Exp Immunol 1992;90:20914.

13 Hull J, Thomson AH. Contribution of genetic factors other than CFTR to disease severity in cystic fibrosis. Thorax 1998;53:1018-21.

14 Gabolde M, Guilloud-Bataille M, Feingold J, et al. Association of variant alleles of mannose binding lectin with severity of pulmonary disease in cystic fibrosis: cohort study. BM7 1999;319:1166-7.

15 Garred P, Pressler T, Madsen HO, et al. Association of mannose-binding lectin gene heterogeneity with severity of lung disease and survival in cystic fibrosis. F Clin Invest 1999;104:431-7.

16 Aron Y, Polla BS, Bienvenu T, et al. HLA class II polymorphism in cystic fibrosis. A possible modifier of pulmonary phenotype. Am $\mathcal{F}$ Respir Crit Care Med 1999;159:1464-8

17 Mahadeva R, Dunn AC, Westerbeek RC, et al. Antineutrophil cytoplasmic antibodies against bacterial/permeability increasing-protein and cystic fibrosis lung disease. Clin Exp Immunol 1999;117:561-7.

18 Döring G, Krogh-Johansen H, Weidinger S, et al. Allotypes of $\alpha_{1}$-antitrypsin in patients with cystic fibrosis, homozygous and heterozygous for in patients with cystic fibrosis, 18:3-7.

19 Mahadeva R, Westerbeek R, Perry DJ, et al. $\alpha_{1}$-antitrypsin deficiency alleles and the Taq-1 G $\rightarrow$ A allele in cystic fibrosis lung disease. Eur Respir 7 1998; 11:873-9.

20 Arkwright PD, Laurie S, Super M, et al. TGF- $\beta_{1}$ genotype and accelerated decline in lung function of patients with cystic fibrosis. Thorax 2000;55:459-62. 